\title{
THE EXTREME ENERGY COSMIC RAYS PUZZLE
}

\author{
A. Pagliaro \\ Istituto di Astrofisica Spaziale e Fisica Cosmica (INAF), Palermo, Italy \\ (Received September 5, 2007; received in final form February 4, 2008)
}

\begin{abstract}
After the discovery of the cosmic microwave background, it was shown that high energy cosmic rays should be seriously attenuated. Nevertheless, several events have been detected. The sources of these cosmic rays and the production mechanisms are still a mystery and the uncertain evidence of the cut-off due to the cosmic background is today's main challenge. Something fundamental seems to miss in our understanding of the nature and propagation of cosmic rays and their sources. How can these particles be accelerated or produced at such energies? Which sources are responsible? Since their origin seems to be extragalactic, how can they travel over cosmological distances? In this paper we see why these questions are still unanswered and what are the most plausible answers so far.
\end{abstract}

Key words: high energy cosmic rays.

PACS number(s): 96.50.S-, 98.70.Sa

\section{INTRODUCTION}

Soon after the discovery of the cosmic microwave background (hereafter CMB), it was shown that cosmic rays (hereafter CRs) with energies in excess of $5 \cdot 10^{19} \mathrm{eV}$ should be seriously attenuated by photopion production due to interaction with the CMB. This effect is known as Greisen-Zatsepin-Kuzmin cut-off (GZK) [1,2]. As a consequence, CRs of high energies should not be able to reach the Earth from cosmological distances.

Nevertheless, several cosmic rays events with energies greater than $E_{\mathrm{GZK}} \sim 5 \cdot 10^{19} \mathrm{eV}$ have been detected by a number of extreme energy cosmic rays observatories (AGASA, Fly's Eye, Haverah Park, HiRes, Auger).

The sources of these extreme energy Cosmic Rays and the production mechanisms are still unknown, and the uncertain evidence of a GZK feature, even if HiRes experiment recently claimed to have observed it, is the main challenge that models for EECRs production and propagation have to face. Moreover, the already mentioned GZK effect should reduce the loss distance for protons and nuclei to a few $10 M p c$ : reaching us would be impossible for extragalactic cosmic rays, while the isotropy of events would favour an extragalactic origin. Besides, according to Wolfendale [3] and Bird [4] at least some of the most energetic CRs are probably protons, so an origin within our Galaxy runs into several problems. The most energetic CRs observed are presently one of the most puzzling problems in astrophysics.

Therefore, something fundamental seems to miss in our understanding of the nature and propagation of cosmic rays and/or their sources. The main questions still waiting for an answer are at least three:

1) How can these particles be accelerated or produced at such high energies?

2) Which are the sources responsible for acceleration/production?

3) Since their origin is likely to be extragalactic, how can they travel undisturbed over cosmological distances?
In the following we will see why these questions are still waiting for an answer and what are the most plausible answers hypothesized so far.

Units useful in the subject and used in the following way: $\mathrm{GeV}\left(1 \mathrm{GeV}=10^{9} \mathrm{eV}\right), \mathrm{TeV}\left(1 \mathrm{TeV}=10^{12} \mathrm{eV}\right)$, $\mathrm{PeV}\left(1 \mathrm{PeV}=10^{15} \mathrm{eV}\right), \mathrm{EeV}\left(1 \mathrm{EeV}=10^{18} \mathrm{eV}\right), \mathrm{ZeV}$ $\left(1 \mathrm{ZeV}=10^{21} \mathrm{eV}\right)$.

\section{FACTS: THE DATA}

Cosmic Rays are elementary particles, nuclei and electromagnetic radiation of extraterrestrial origin. When a single high energy cosmic ray particle hits the upper atmosphere, an extensive air shower develops as a result of interactions. The secondary particles produced in each collision may either decay or interact with another particle, thereby multiplying the particles. After reaching a maximum the shower attenuates as more and more particles fall below the threshold for further production. A disk of particles extended over an area of some tens of meters at $10^{14} \mathrm{eV}$ can then be observed at ground. By means of measurements on the secondary particles and the Cherenkov radiation we can determine the parameters of the progenitor. This determination is, however, very difficult, due to the stochastic nature of the huge numbers of the interaction.

Probably the most difficult parameter to be determined is the nature of the progenitor: whether it is proton, photon, helium or another nucleus. However difficult it may be, determining the nature is of great importance for establishing the mass composition of cosmic rays. Theoretical models predict a change in composition beyond the knee, so that determination of the elemental composition in cosmic rays initiated showers is crucial for model constraining (e.g. [54]).

A few techniques are in use for determining the nature of the progenitor. The muon content is used in separat- 
ing gamma ray from cosmic rays initiated showers [55]. Various parameters of showers (lateral distribution of the Cherenkov light [56], lateral distribution of charged particles in the shower core [4], location of the shower maximun [58]) have also been used in shower separation and determination of the elemental composition of cosmic rays.

So far, we have observed cosmic rays in the energy range $10^{9} \mathrm{eV}$ up to $3.2 \cdot 10^{20}$. In this range, the flux follows a broken power law with indices -2.7 and -3.1 . Remarkably, it appears to be a smooth curve over 10 decades of energy with only a few noticeable structures. The most prominent of these are small, abrupt changes in the spectrum index. Only two of these changes are clearly visible in the spectrum: the first corresponds to energy $10^{15} \mathrm{eV}$, the second at about $5 \cdot 10^{18}$. In an antropomorfic view of the spectrum, the two structures are known as the knee and the ankle (Fig. 3)

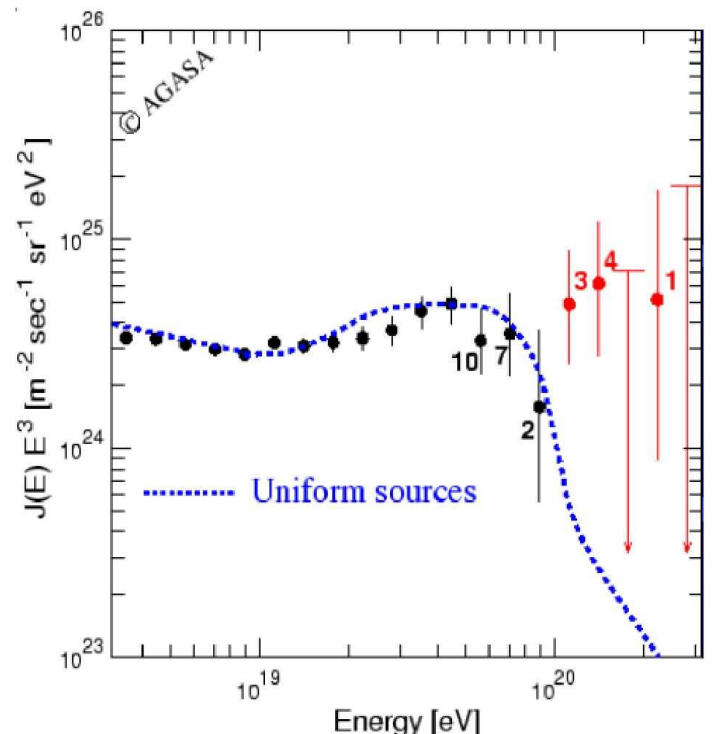

Fig. 1. EECRs spectrum as observed by AGASA. The dashed line is the spectrum expected from uniformly distributed astrophysical source (from the AGASA website).

Cosmic Rays with energies greater than the ankle are known in the literature as Extreme Energy (or Ultra High Energy) Cosmic Rays (EECRs or UHECRs, we will use the former in the following). Their mere existence poses a serious challenge to the Standard Model of contemporary Physics.

In October 1991, the Fly's Eye cosmic ray detector recorded an event of energy $\sim 320 \mathrm{EeV}$. This is the highest energy event recorded so far. In fact, all experiments have recorded cosmic rays in the vicinity of $100 \mathrm{EeV}$. However, how well experiments can determine the energy of these events is a critical issue. All techniques used are subject to ambiguity of particles simulations which involve physics beyond Large Hadron Collider limits.

Figure 1 shows the data on the EECRs spectrum from Akeno detectors (AGASA). Other data from Fly's Eye, Havera Park and Yakutsk available [5] are rather consistent with Figure 1.

Nowadays, the most significant data are those collected by AGASA, HiRes and Auger. AGASA and HiRes have discrepant results in the highest energy part: AGASA does not show GZK suppression, while HiRes does. However, recent simulations of De Marco, Blasi and Olinto [61] show that a systematic error of $30 \%$ would make the two experiments reasonably agree with each other.

The most recent measurement by Auger [62] is in closer agreement with the HiRes results. However, no conclusive evidence for the absence of the GZK feature can be claimed so far.

EECRs data in a nutshell are:

- Most energetic event: Fly's Eye (10/1991): 320 EeV

- Volcano Ranch (1962): 1 event above $100 \mathrm{EeV}$

- Haverah Park (1987): 4 events above 100 EeV

- AGASA (1990-2004): 11 events above $100 \mathrm{EeV}$

- HiRes Fly's Eye (1999-2006): 7 events above 100 $\mathrm{EeV}$

- Pierre Auger Obs. (2004-2007): 27 events above 57 $\mathrm{EeV}$

In 2006 AGASA result was retracted. They discovered an error in data analysis, so that their highest energy events should be lowered by $15 \%$. The result reduces the 11 events to about 5 or 6 .

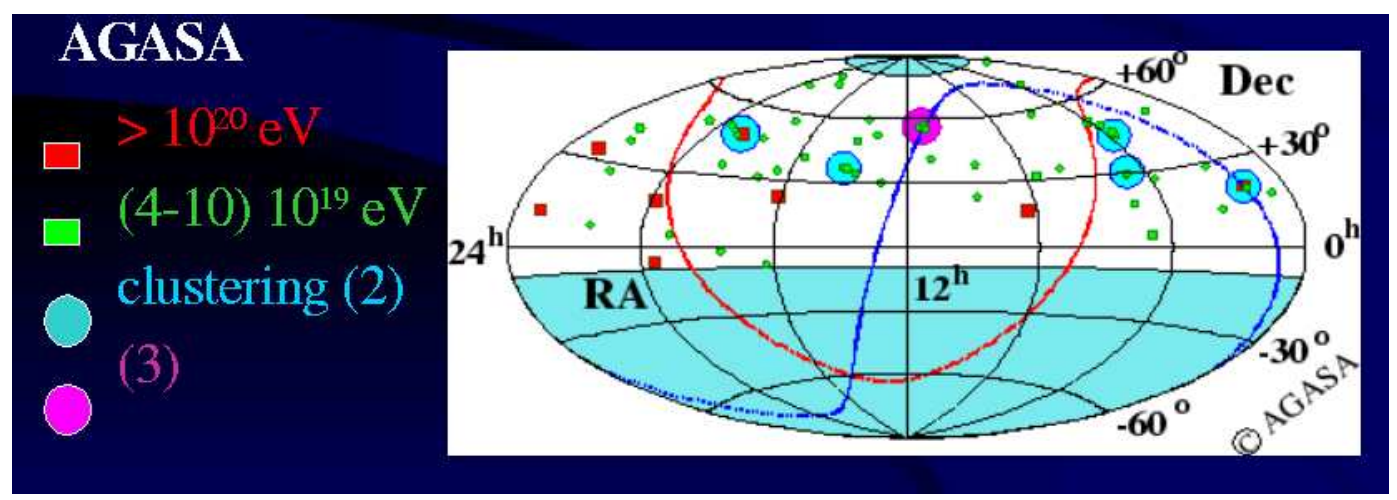

Fig. 2. Cosmography: the map shows clustered events (doublets and a triplet) as seen by AGASA. 


\section{A. Cosmography}

The arrival directions of EECRs seem to be isotropic. According to present AGASA data, EECRs do not see the Galaxy, the Local Group, the Local SuperCluster. There is presently no clear evidence of correlations between the arrival directions of the events and any of the known luminous structures (however, we should always keep in mind that above $100 \mathrm{EeV}$ we have few events).

The apparent isotropy (no galactic plane enhancement) of cosmic rays above $10 \mathrm{EeV}$ [6], together with the difficulty of confining protons in the galaxy at 10 to $30 \mathrm{EeV}$, provide significant reasons to believe that cosmic rays above $10 \mathrm{EeV}$ are extragalactic in origin.

\section{B. Correlations}

Recently, the Auger team finds [11] that cosmic rays with energy higher than $57 \mathrm{EeV}$ (Auger sees 27 events) may come from directions within 3 of AGNs. The cosmic rays do not point precisely to the AGNs: the galactic magnetic field may deflect them. This result do not obviously prove that AGNs are the sources: anything else distributed on the sky in the same way as AGNs could be.

Still, not everyone is convinced that the observation will hold up as Auger collects more data. Even with the check against the second data set, the Auger team estimates that there is a $0.1 \%$ chance the correlation with AGNs is meaningless.

Apart from AGNs, there is no other evidence for correlations with known celestial objects. A weak correlation with quasars and BL Lac objects has been under debate since a few years ago. Also a weak correlation with clusters of galaxies (in particular with Virgo, Coma, AWM7) is possible. However, present evidence is very weak.

\section{Composition}

In the energy range from $10^{12}$ to $10^{15} \mathrm{eV}$ CRs arriving at the edge of the atmosphere have been measured to consist of $\sim 50 \%$ protons, $\sim 25 \%$ alpha particles, $\sim 13 \%$ $\mathrm{C} / \mathrm{N} / \mathrm{O}$ nuclei, $\leq 1 \%$ electrons, $\leq 0.1 \%$ gammas.

At higher energies, measurements are much more difficult and ambiguous and events are rare.

However, at EeV energies the primary particles appear to have a mixed or heavy origin, probably trending toward a protonic origin in the higher energy range around $30 \mathrm{EeV}[8,9]$. This trend, together with evidence of a flattening in the cosmic ray spectrum on the 3 to $10 \mathrm{EeV}$ energy range [4] gives evidence for a new component of cosmic rays dominating above $10 \mathrm{EeV}$.

The EECRs chemical composition is very likely to be unveiled only on a statistical basis. Our present knowledge is weak and controversial due to the limited events. Fly's Eye data show evidence of a shift from a dominantly heavy composition (maybe iron) to a light one (maybe protons). AGASA's identification is based on muon content. Initially their result was quite the opposite: no change in composition. However, a recent review of both methods [10] has shown that the inconsistencies were mainly due to the scaling assumption of the interaction model used by AGASA. The authors conclude that if a different model is used both data sets demonstrate a change in composition: a shift from heavy (at $0.1 \mathrm{EeV}$ ) to light (at $10 \mathrm{EeV}$ ).

Gamma rays also have high cross sections with air and are yet another candidate but no evidence was found up till now for a gamma signature among the higher energy events. The $320 \mathrm{EeV}$ event was studied in detail and found incompatible with an electromagnetic shower [12].

\section{LOW ENERGIES: A SOLVED PROBLEM?}

Sometimes it seems that the question of low-energy cosmic ray origin was some old problem left over from the last century and recently has been solved by the Biermann SNRs theory [13]. Probably, nothing is further from the truth. Cosmic ray origin is the only important physics problem where astroparticle physics is called upon to take the lead in providing a full solution for all energies [14].

In fact, in spite of enormous advances in experimental techniques, not a single additional observational fact directly supporting the present paradigma that SNRs are the major source of Galactic cosmic rays has come to light.

To make matters worse, it has been recently learnt that Cas A is not an important source of hadronic cosmic rays. As a matter of fact, we do think that a unitary theory would be important for at least three reasons:

1) A power law with index -3 is a good approximation for all the spectrum;

2) There is no clear cut-off in the spectrum;

3) The spectrum is very smooth even at the joints between different regions, while these should be explained by different theories/processes that would not necessarily produce smooth transitions in the spectrum.

This evidence does not find any justification in present "eclectic" theories that invoke a different process for each region of the spectrum. A unitary theory would be much more simple and definitely able to inspire the sense of inevitability typical of the most "beautiful" theories of Physics.

We are not going to write more about low energies. But we believe that the low energies argument cited should, however, be considered a caveat when introducing new theories trying to explain CRs spectrum.

\section{THE PROPAGATION}

\section{A. The GZK Effect}

In 1965, Penzias and Wilson [15] reported the discovery of the cosmic $3 \mathrm{~K}$ thermal blackbody radiation (the 
CMB) which was produced in the early history of the Universe. More recently, COBE [16] and WMAP [17] experiments confirmed the discovery showing that the radiation is also isotropic to a part in $10^{5}$. The radiation is cosmological and fills the entire Universe with a $2.7 \mathrm{~K}$ spectrum of photons with a density of about $400 \mathrm{ph} / \mathrm{cm}^{3}$.

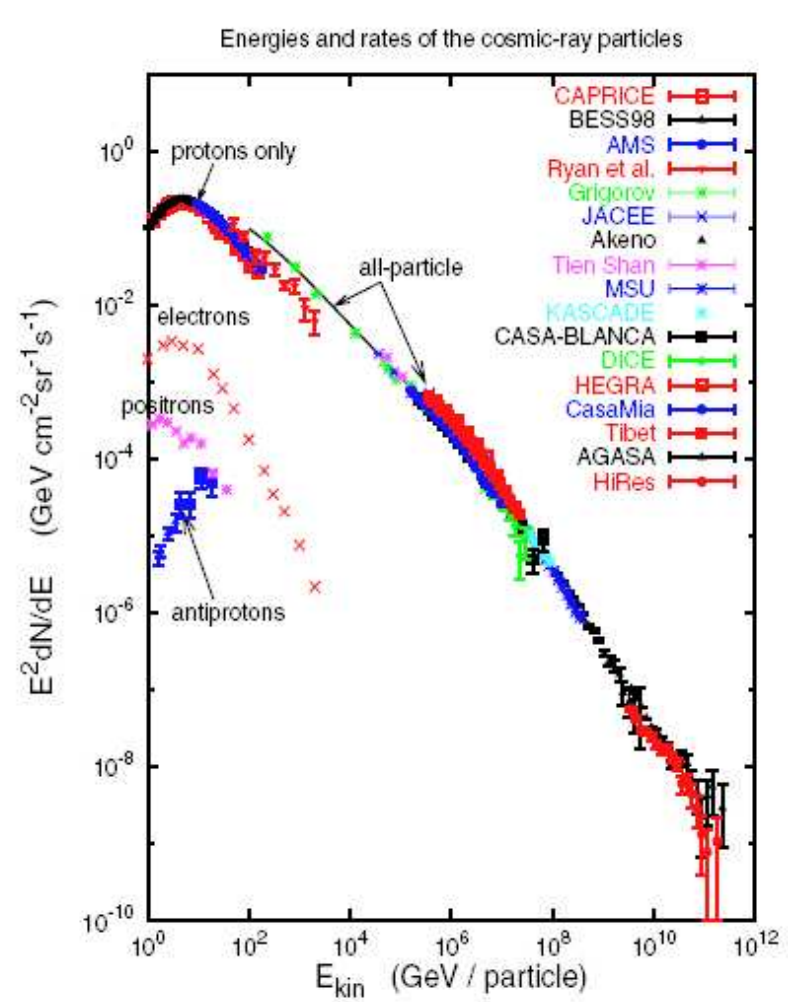

Fig. 3. Cosmic rays spectrum showing the two features known as knee and ankle (from Gaisser [59]).

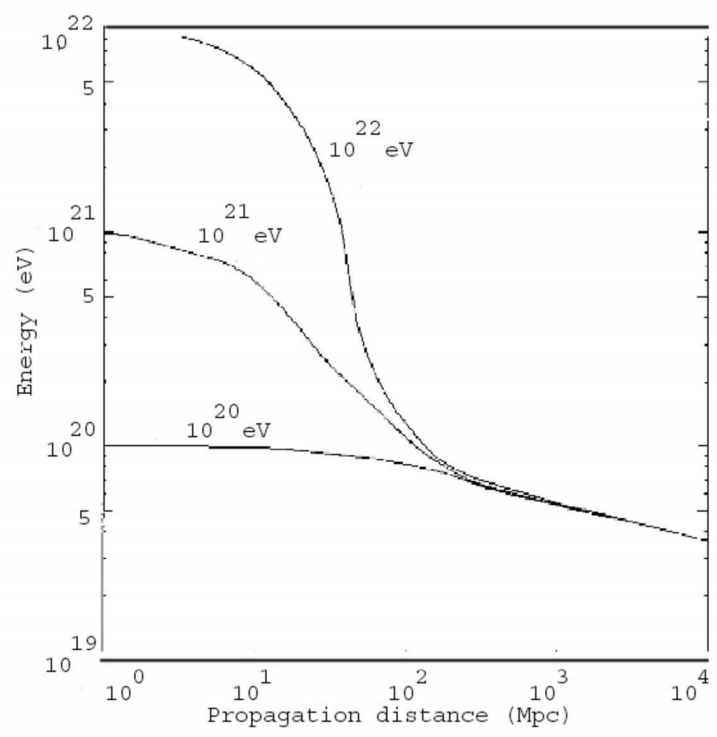

Fig. 4. Energy loss during propagation for a proton with a given initial energy: a proton of $200 \mathrm{Eev}$ has a mean life of $3.0 \cdot 10^{15} \mathrm{sec}$ or $30 \mathrm{Mpc}$, a proton of $300 \mathrm{EeV}$ has a mean life of $1.5 \cdot 10^{15} \mathrm{sec}$ or $15 \mathrm{Mpc}$.
The interactions between CMB photons and CRs are of four kinds:

1) Compton interaction of nuclei. The energy loss is negligible and this process will not be considered in the following;

2) Pair production:

$$
\begin{aligned}
& p+\gamma_{2.7 \mathrm{~K}} \rightarrow p+e^{+}+e^{-} \\
& A+\gamma_{2.7 \mathrm{~K}} \rightarrow A+e^{+}+e^{-}
\end{aligned}
$$

Theoretical energy loss for protons is about $0.1 \%$, while real energy loss is considerably larger (see fig.9);

3) Photodisintegration of the nucleus:

$$
\begin{aligned}
& A+\gamma_{2.7 \mathrm{~K}} \rightarrow(A-1)+N \\
& A+\gamma_{2.7 \mathrm{~K}} \rightarrow(A-2)+2 N
\end{aligned}
$$

4) Photopionic production:

$$
\begin{aligned}
& p+\gamma_{2.7 \mathrm{~K}} \rightarrow \Delta^{+} \rightarrow n+\pi^{+} \\
& p+\gamma_{2.7 \mathrm{~K}} \rightarrow \Delta^{+} \rightarrow p+\pi^{0}
\end{aligned}
$$

and at higher energies:

$$
\begin{gathered}
p+\gamma_{2.7 \mathrm{~K}} \rightarrow \Delta^{++}+\pi^{-} \\
\Delta^{++} \rightarrow p+\pi^{+}
\end{gathered}
$$

with theoretical energy loss for proton about $20 \%$, while real energy loss is larger (see fig. 9).

Shortly after the CMB discovery, Greisen [1] and Zatsepin and Kuzmin [2] predicted that the forth and most important process, the pion-producing interactions of ultrahigh energy cosmic ray protons with CMB photons, should produce a cut-off in their spectrum at energies greater than $50 \mathrm{EeV}$. This effect has since then become known as the GZK effect.

The actual position of GZK cut-off can actually somewhat differ from the $50 \mathrm{EeV}$ predicted by them. In fact, there could be an enhancement at or near this energy owing to a "pile-up" of cosmic rays starting out at higher energies [18]. Needless to say, the existence of the "pileup" not yet clearly revealed by any experiment would be evidence for the GZK effect.

Talking about the GZK effect, we focus on the propagation of atomic nuclei (in particular protons) and photons. Electrons are not considered as possible EECRs because they radiate most of their energy while crossing the cosmic magnetic fields. Among the known particles, nuclei and neutrinos are the only possible candidates. Neutrinos should deserve special attention as they may travel through space almost unaffected. However, the interaction of neutrinos should occur uniformly in the atmosphere, a feature not reproduced by current data. While 
neutrinos may be one of the components of extreme energy end of the spectrum, they do not seem to dominate at least up to energies of a few $10^{20} \mathrm{eV}$.

The numbers: a simple computation shows that the GZK cut-off threshold for collisions between the CMB and protons (the so-called process of pion photoproduction, process $n .4$ above), for an energetic CMB photon with a common value of $\epsilon=10^{-3} \mathrm{eV}$, is $7 \cdot 10^{19} \mathrm{eV}$ which is where we expect the cut-off to start.

The interaction length for the process can be estimated from the photopion production cross-section and from the $\mathrm{CMB}$ photon density and is about $6 \mathrm{Mpc}$ for the values $\rho_{\mathrm{ph}}=410 \mathrm{~cm}^{-3}$ and $\sigma=135 \mu$ barns. Above $100 \mathrm{Mpc}$ of propagation distance, the observed energy of protons is below $10^{20}$ regardless of the initial value (see Fig. 4). The probability to travel without losses is negligible.

A more careful calculation shows that the GZK cut-off is not an absolute end to the cosmic ray spectrum but it should give rise to a clear feature around the energy $5 \cdot 10^{19} \mathrm{eV}[19]$. The local distribution of sources can significantly affect the agreement between predicted and observed spectra [19]. In principle, a local overdensity of sources can decrease the gap between observed and detected post-GZK events. A local overdensity will increase the observed flux at the highest energies relative to the lower energy flux. If the EECRs source distribution is proportional to that given by the galaxy distribution the observed local overdensity is not high enough to explain the data.

For nuclei, the situation is more difficult. They undergo photodisintegration in the $\mathrm{CMB}$ and infrared radiation losing on average 3 to 4 nucleons per Mpc when their energy exceeds $2 \cdot 10^{19} \mathrm{eV}$. However, the IR background is much less known than the CMB and the attenuation length for nuclei must be handled with precaution.

One could say: there could be some sources close to us that the observed spectrum does not suffer the cutoff. But the problem is the low energy part: it is formed by sources at large distances and because of GZK these sources cannot contribute to the high energy part. If the assumption about distribution of sources is uniformity, then a serious problem arises.

Recently, the HiRes experiment claimed to have observed GZK cut-off using the fluorescence techniques together with an augmented Monte Carlo simulation method [53]. As said before, recent Auger data [62] are in agreement with the HiRes results but a conclusive evidence for the absence of the GZK feature cannot be claimed.

\section{ASTROPHYSICAL ZEVATRONS}

The mystery presented by the data above $10^{20}$ has given rise to a growing number of proposals. It is not surprising that the number of proposals discussed in the literature is very likely higher than the number of postGZK events observed. A first approach to the proposals can be done dividing them into two big areas: the Astrophysical Zevatrons or Bottom-Up models and New
Physics (mainly Top-Down models). The former involves searching for acceleration sites in the known astrophysical objects that could reach $\mathrm{ZeV}$ energies, while the latter includes hybrid and top-down models and involves extensions of the Standard Model.

Astrophysical Zevatrons are also referred to as bottomup models: they are known astrophysical objects that can reach energies of the order $\mathrm{ZeV}\left(1 \mathrm{ZeV}=10^{21} \mathrm{eV}\right)$. Cosmic rays can be accelerated in these objects when large-scale macroscopic motions are transferred to microscopic particles. Essentially, two types of acceleration mechanism are distinguished:

- Direct one-shot acceleration by high electric fields. This occurs in very compact objects such as highly magnetized neutron stars or accretion discs of black holes. This mechanism does not provide a natural power-law spectrum;

- Diffusive stochastic shock acceleration in magnetized plasma clouds which generally occurs in all systems where shock waves are present (e.g. SNRs or filamentary structures around clusters of galaxies). This statistical acceleration is known as the Fermi mechanism of first or second order (see Fig. 5 and 6$)$.

This second kind of mechanism is the one which could be responsible for EECRs acceleration in Zevatrons. We are not interested here in the details of the acceleration mechanisms. We refer the interested reader to Protheroe [20] or Baring [21].

The Fermi mechanism has the great advantage of transforming the problem: from a "how is it possible" problem to a "where is it possible" one.

\section{A. The Hillas Plot}

It has become quite popular to accept or rule out EECRs bottom-up production sources by means of a Hillas plot [22], that is as a matter of fact the standard rule to accept/reject any kind of source inside the number of candidate Zevatrons. According to this constrain, the maximum energy of accelerated particles can be estimated by requiring that the gyroradius of the particle be contained in the acceleration region. This condition in its simplest form reads as:

$$
E_{\max }=Z(B L)
$$

where $\mathrm{E}$, in $\mathrm{EeV}$, is the maximum energy available in the source for accelerating particles, B, in $\mu G$ is the mean magnetic field inside the source and $\mathrm{L}$, in $\mathrm{kpc}$, is the typical scale of the source.

This assumes that particles can be accelerated up until the moment when they can no longer be confined inside the source.

Inspecting the Hillas diagram, one notices that only a few astrophysical sources satisfy the necessary (but clearly not sufficient) condition. We explore them in more details below, not before having reminded to the reader two 


\section{A. PAGLIARO}

more additional constraints that should be verified in the sources.

\section{B. Additional constraints}

In addition to the Hillas constrain, candidate sources must also satisfy at least two additional conditions:

- The acceleration must occur in a reasonable time scale, e.g. the size of the acceleration region must be less than the interaction length of the accelerated particle. The rate of energy loss on the CMB must be less than the rate of energy gain;

- The acceleration region must be large enough so that synchrotron losses are negligible if compared to the maximum energy of Hillas formula.

We explore now in more details the most plausible candidate Zevatrons.

\section{Candidate Zevatrons}

\section{Pulsars}

From a dimensional analysis, the electric field potential drop in a rotating magnetic pulsar could be as high as $e \Delta \Phi=100 \mathrm{EeV}$. However, the high radiation density in the vicinity of the pulsar will produce $e^{+} e^{-}$pairs from conversion in the intense magnetic field. [23] These pairs will drift in opposite directions along the field lines and short circuit the potential drop. A more realistic approach further decrease the initial estimate. Moreover, synchrotron radiation losses in such compact systems become very important even for protons.

\section{AGN Radio Lobes}

On the list of plausible Zevatrons are also powerful radio galaxies. Jets from the central black-hole of an active galaxy end at the termination shock where the interaction of the jet with the intergalactic medium forms radio lobes and hot spots. Of special interest are the most powerful AGNs where shocks can accelerate particles to energies above EeV via Fermi mechanism. These sources may be responsible for the flux of EECRs up to the GZK cutoff. [24] A nearby specially powerful source may be able to reach energies past the cutoff. However, extremely powerful AGNs with hot spots are rare and far apart.

\section{AGN Central Regions}

The powerful engines that give rise to the observed jets and radio lobes are located in the central regions of active galaxies and are powered by the accretion of matter onto supermassive black holes. It is reasonable to consider the central engines as likely accelerators. In principle, the nuclei of generic active galaxies can accelerate via a unipolar inductor. In the case of AGNs, the magnetic field may be provided by the infalling matter. The spinning black hole horizon provides the imperfect conductor. The problem as EECRs sources is twofold: first, EECRs face debilitating losses in the acceleration region due to the intense radiation field, second the spatial distribution of objects should give rise to a GZK cut-off in the spectrum.

\section{Fanaroff-Riley Class II Objects}

Radio loud quasars are characterized by a very powerful central engine ejecting matter along this extended jets. At the ends of those jets (hot spots), the relativistic shock might be able to accelerate particles up to $\mathrm{ZeV}$ energies. FR-II galaxies seem the best potential astrophysical sources for EECRs. [25] Unfortunately no nearby objects of this type are visible in the direction of observed EECrs events. The closest FR-II source is at about $2.5 \mathrm{Gpc}$, even if in the direction of the $320 \mathrm{EeV}$ event.

\section{Gamma Ray Burst}

GRB are an intense source of gamma rays of a few milliseconds with gamma energies ranging from $1 \mathrm{KeV}$ to a few $\mathrm{GeV}$. Several thousands have been observed. GRBs and EECRs have similarities that may argue for a common source. GRBs are distributed isotropically and the average rate of $\gamma$ ray energy emitted by GRBs is comparable to the energy generation rate of EECRs of energy $>10^{19} \mathrm{eV}$ (in a redshift independent cosmological distribution of sources).

However, the distribution of EECRs arrival directions and arrival times argues against the common origin. Events post-GZK require contribution only from GRB inside a $50 \mathrm{Mpc}$ radius. Since less than one burst is expected within this region in $100 \mathrm{yr}$, the unique source would appear as a concentration of EECRs events in a small part of the sky, and the signal should be narrow in energy.

\section{Neutron Stars}

Neutron stars have the ability to confine $\sim 10^{20} \mathrm{eV}$ protons and they can also match the observed EECRs fluxes. However, ambient magnetic and radiation fields induce significant losses inside the light cylinder. Only the plasma out of the cylinder can be accelerated to extreme energies. In this case, EECRs originate mostly in the Galaxy and the primaries should be heavy nuclei. This proposals should be constrained once the primary composition is clearly determined. 


\section{Regions of intense star formation}

$\mathrm{TeV} \gamma$-rays may provide significant information about astrophysical Zevatrons. In 2002, the HEGRA [29] collaboration detected gamma rays coming from the core of Cygnus OB2, a region of intense star formation. The gamma rays have $\mathrm{TeV}$ energies, but no corresponding radio, optical, or X-ray emission come from the same location. Although standard models for $\gamma$-ray production are two (first, purely electromagnetic: highly-accelerated electrons bang into low-energy photons, bumping them up to gamma-ray energies, second, termed hadronic: collisions between high-speed protons and low-energy protons or photons create neutral pions that eject gamma rays when they decay), neither works well for Cygnus OB2. The electron collision mechanism would produce detectable X-rays, while the proton collision mechanism requires higher gas densities or higher proton energies than Cygnus OB2 likely possesses. In a recent paper Anchordoqui et al. [27] present a third dynamic. The model proposes that the observed $\mathrm{TeV} \gamma$-rays are the result of Lorentz boosted $\mathrm{MeV} \gamma$-rays emitted on the de-excitation of daughter nuclei following collisions of $\mathrm{PeV}$ nuclei with a ultraviolet photon background. In other words, a nucleus with sufficient speed absorbs a photon and is excited into a giant dipole resonance. Then, the nucleus decays and emits a photon. This mechanism predicts a drop-off in $\gamma$-ray emission below $1 \mathrm{TeV}$ that future observations could verify. What is important to us, however, is that this model suggests that regions of intense star formation and dense stellar radiation like Cygnus OB2 could also be the sites of cosmic ray acceleration. That is because the same wind-swept nuclei that power the $\gamma$-ray emission would also stream away into the galaxy at large, and some would eventually bombard Earth as cosmic rays.

Recently, also HESS collaboration has spotted $\gamma$-ray emission of very high energy from the powerful young stellar association Westerland 2 [30]. The $\gamma$-rays detected have energies in the range of $\mathrm{TeV}$. The HESS finding seem to confirm the viability of turbulent - Fermi II as the mechanisms for cosmic rays acceleration. In this respect, it would be useful to develop the details of the superbubble model [31] for the acceleration of cosmic rays, so that concrete predictions could be made.

\section{Large Scale Structure and Cosmological Voids}

It is now believed that CRs are diffusively accelerated at many shocks by the I order Fermi mechanism. [26] The strongest and largest shocks in the Universe are accretion shocks around clusters of galaxies. The maximum energy at which a particle can be accelerated inside these structures is controversial. However, cluster shocks, although very large, seem not to be able to accelerate protons to energies above $\sim 10^{20} \mathrm{eV}$.

Moreover, CRs accelerated inside or around large scale structures such as clusters of galaxies would probably be unable to get out and reach the observer, due to photopion production inside the cluster: as a matter of fact, propagation inside the cluster generates a GZK feature.

In a recent paper Antonuccio et al. [32] propose a possible way out: cosmic rays could propagate more easily inside cosmological voids than in cosmological background. In the 1970s a series of studies showed the existence of filamentary distribution of galaxies in clusters and superclusters and large void regions among such filaments. These regions are known as Cosmological Voids. For example, a redshift survey in the Bootes region [33] showed a large void structure whose estimated diameter is $60 h^{-1} \mathrm{Mpc}$. From a theoretical-simulative point of view, voids are easily formed in Universes simulated by means of N-body codes.

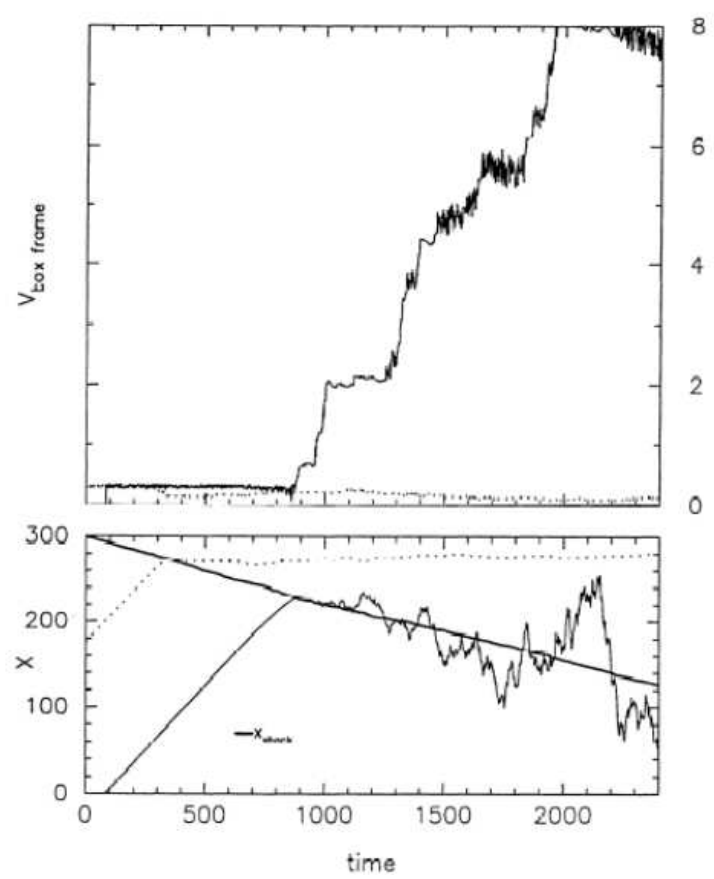

Fig. 5. Position and velocity vs. time for two particles inside a shock. The lower panel shows the position of the shock (thick line) and two particles, the dotted line traces a particle that represents the majority of particles, the solid line traces a particle that is injected into the Fermi acceleration process. The top panel shows each particle's corresponding total velocity (from Knerr [63]).

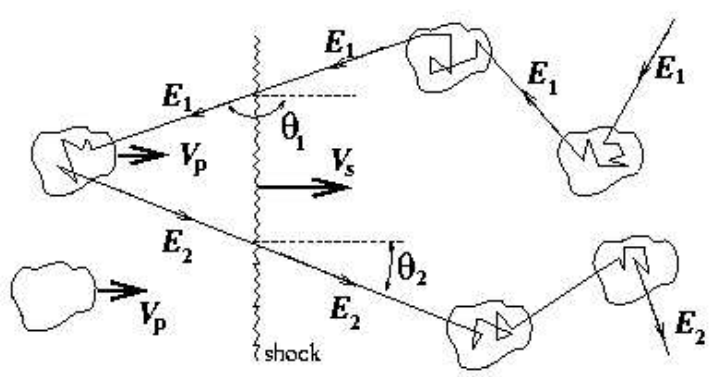

Fig. 6. Interaction of cosmic ray of energy $E_{1}$ with a shock moving with speed $V_{s}$ (from Protheroe [20]).

Inside these void regions, life is easier for cosmic rays and they should propagate suffering a reduced GZK ef- 
fect. This is because $E_{\mathrm{GZK}}$ depends on the average Cosmic Microwave Background photon energy and the loss distance $\left(d_{\text {loss }}=E d r / d E\right)$ depends on the CMB energy density. Even if fluctuations in the CMB energy density are very small, we have shown [32] that this effect is very sensitive to fluctuations and could be not negligible and precise computation of EECRs fluxes for future experiments should take it in account.

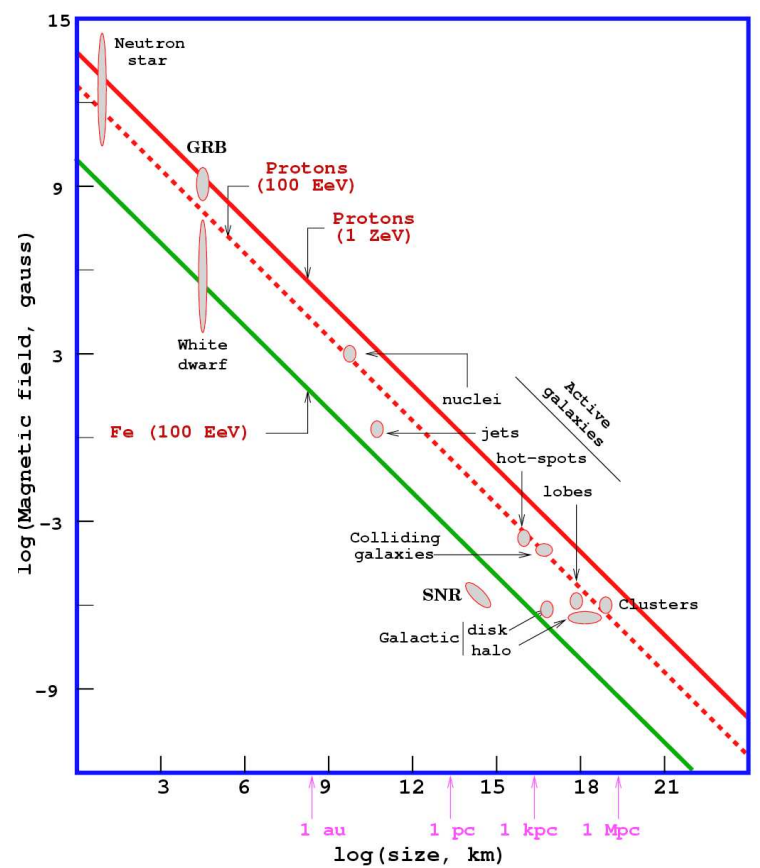

Fig. 7. The Hillas Plot (from the Universität of Erlangen-Nürnberg website).

The model is as follows: EECRs are probably produced in shocks along filaments and walls (but they actually could be produced in any other model, be it top-down or bottom-up) in the neighbourhood of a Cosmological Void (or inside). At some point in their propagation history, EECRs enter voids. Here they are confined by a (possible) large scale magnetic field inside and they are bounced back when reaching the walls. Inside the void (especially in the central region), they propagate almost freely suffering a reduced GZK effect. At the walls, they can be furtherly accelerated by some Fermi I mechanism.

\section{Being conservative: heavy nuclei}

The most conservative hypothesis for explaining the post-GZK effect is probably the iron hypothesis. That is: the post-GZK events are produced by heavy nuclei. In 1999 Stecker and Salamon [34] have shown that the energy loss time for nuclei starting out as $\mathrm{Fe}$ is longer than that for protons for energies up to a total energy of $300 \mathrm{EeV}$. An interesting clue that we may be seeing heavier nuclei comes from a very recent analysis of inclined air showers above $10 \mathrm{EeV}$. [35] These results favor proton primaries below the GZK cutoff but favor a heavier composition above. Fe nuclei could have a de- layed GZK cutoff. The details are complicated but the relevant quantity in the problem is $\gamma=E / A M$ where $\mathrm{A}$ is the atomic number and $\mathrm{M}$ is the nucleon mass. For a fixed observed energy, the smallest boost above GZK threshold is associated with the largest atomic mass (see Fig. 9).

\section{NEW PHYSICS}

The astrophysical problem is so daunting that many believe that cosmic rays are not the beam of cosmic accelerators, but the decay products of remnants from the early Universe or anything else involving new Physics.

New Physics models can be hybrid or pure top-down models. The former involve Zevatrons and extensions of the particle physics standard model, while the latter involve the decay of very high mass relics from the early Universe and Physics beyond the Standard Model.

A confortable way to avoid the problems of finding plausible zevatrons is to start at the top, i.e. at the energy scale associated with grand unification, supersymmetric grand unification or its string theory equivalent: top-down involves the generation of high energy particles by means of the decay of very massive particles.

The main signatures for Top Down models are:

- Composition: the fragmentation leads to photons ten times more numerous than proton

- Spectrum: QCD fragmentation leads to a nonpower law spectrum

- Cosmography: most likely a halo distribution

\section{A. Topological Defects}

Cosmic Topological Defects are predicted to form in the early Universe as a result of symmetry breaking phase envisaged in unified theories of elementary particle interactions. In a single line, the idea behind topological defects theories is the following:

Topological Defects $\rightarrow$ X Particles $\rightarrow$ Quarks (and Leptons $) \rightarrow$ Hadronization

So, a topological defect will suffer a chain decay into GUT particles $\mathrm{X}$ that subsequentely decay to familiar weak bosons, leptons and quark or gluons jets. Cosmic rays are the fragmentation products of these jets.

The main conditions to be satisfied in order for this to be possible are:

1. X particles should decay in a recent epoch;

2. $\mathrm{X}$ particles mass should be larger than $10^{20} \mathrm{eV}$;

3. The density of topological defects which give origin to the $\mathrm{X}$ particles and their decay rate should be high enough to justify the present flux of cosmic rays. 
Luckily, ZeV energies are not a challenge in a top-down model: symmetry breaking scales at the end of Inflationary Universe are $\gg 10^{20} \mathrm{eV}$ (GUT energy is in fact $\left.10^{24-25} \mathrm{eV}\right)$. Energy greater than $10^{20} \mathrm{eV}$ is easy in topdown models (usually start at $10^{23} \mathrm{eV}$ or higher).

The challenge is the observed flux: the dynamic of the generation and evolution of topological defects from which X particles should originate choose as typical distance among defects the present horizon scale, that is $c H_{0}^{-1} \simeq 3 h^{-1} \mathrm{Gpc}$.

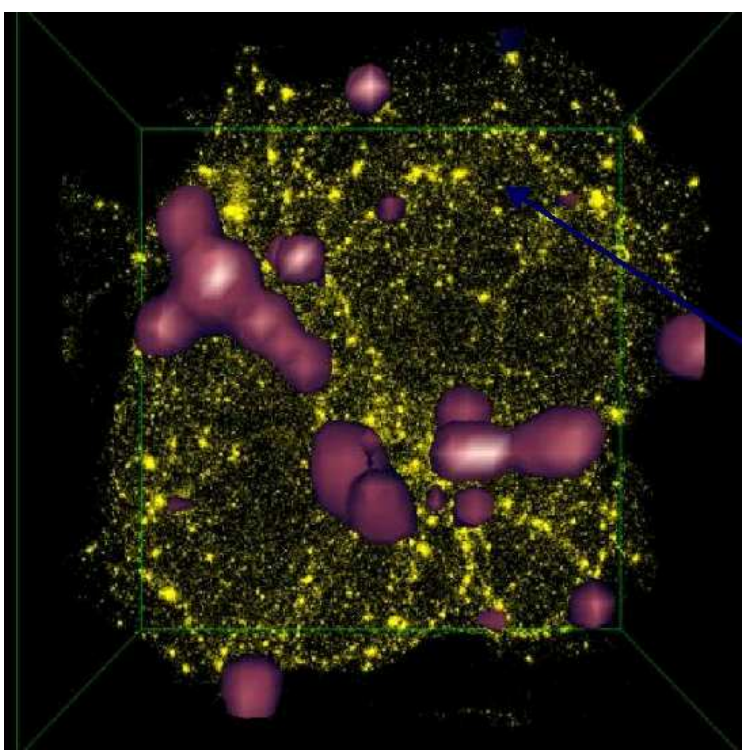

Fig. 8. A simulated box of Universe (side $50 h^{-1} \mathrm{Mpc}$ ) showing clustered structure and an empty region (void) surrounded by filamentary regions (simulation by Antonuccio et al. [32].)

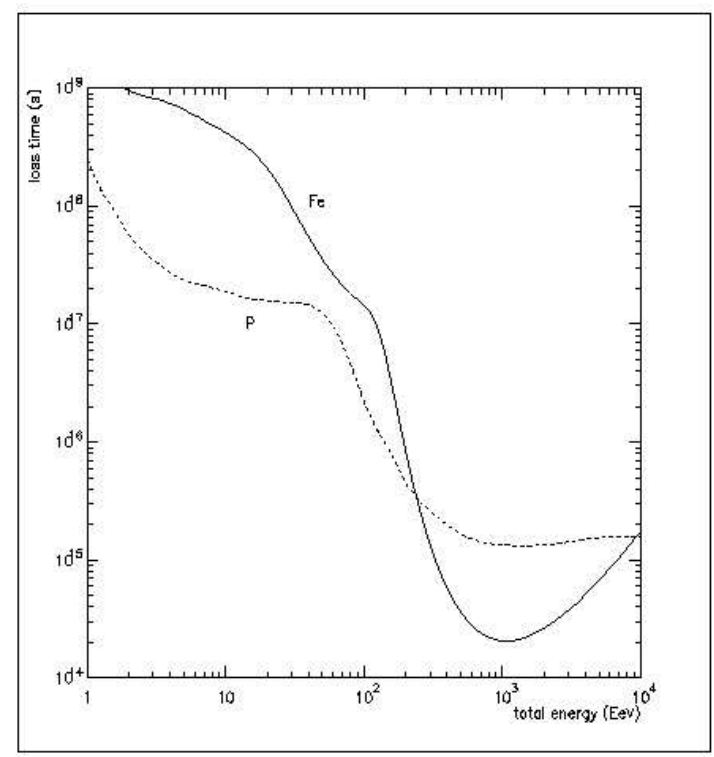

Fig. 9. Comparison of the loss time against total energy for a proton and for an iron nucleus (from Stecker [45]). Adiabatic losses due to redshift are not included.
Moreover and more problematicly, we know from accelerator studies that - among the fragmentation products of jets - neutral pions (decading into photons) dominate protons by two orders of magnitude. Therefore if the decay of topological defects is the source of the highest events, they must be photons. This is presently a problem because the highest event (the $320 \mathrm{EeV}$ one) is not likely to be a photon. [36] The measured shower profile of the event does not support the assumption that the event comes from a photon.

The Topological Defects should be produced at the end of inflation in the early Universe through the Kibble Mechanism as symmetries are broken and decay in a recent epoch or today producing X-particles [37].

Main models of Topological Defects which deserve to be cited are

- Super Conducting Strings [38].

- Cosmic Strings [39]. One way of getting X particles from cosmic strings is through the so-called cusp evaporation mechanism [60]. However, the resulting particle production rate turns out to be too low to be relevant to EHECR. Another possibility arises from oscillation and gravitational radiation, then energy loosing and shrinkage. The resulting flux is again too low. In brief: cosmic strings and superconducting strings are too far apart and the flux seems too low.

- Monopolia [40]. If monopoles were formed in the early Universe, a metastable monopoleantimonopole bound state would be formed. We call this state monopolonium. The efficacy of the collapsing monopolia process depends on two parameters: the monopolonium to monopole fraction at formation and the monopole abundance. The latter is unknown, while computing the first makes monopolia scenario an attractive scenario. Detailed numerical simulations would be useful.

- String Cusps [41].

- Monop-String Network [42].

- Cosmic Necklaces [43]. A cosmic necklace is a possible hybrid topological defect made of a cosmic string with monopole beads. The efficacy of the $\mathrm{X}$ particles production process depends on some free parameters. If these are appropriately chosen, required flux can be obtained.

- Vortons [44].

\section{B. Super Heavy Relic Particles}

The homogeneity of our present Universe may imply an inflationary period in its early history. This phase can lead to a production of extreme energy neutrinos. The inflation is assumed to be controlled by a vacuum field, the inflaton field. The oscillations of the inflaton field reheat the cold post-inflationary Universe to high 
temperatures. In the oscillating field, non-thermal production of very heavy particles (the so-called "wimpzillas") may take place. These particles may have survive of till the present epoch as a part of the dark matter. Their decays would produce extreme energy cosmic rays and photons by means of a fragmentation process. Such particles could be the source of the observed events [45]. If produced in a dark matter halo of our galaxy, nucleons would not be attenuated by GZK effect.

Also this kind of model suffers from a missing photons problem.

\section{Massive Neutrinos}

Turning to the possibility that the primaries may be neutrinos, they should point back to their sources, thereby enabling point-source astronomy for the most energetic sources of flux at and above $10^{20} \mathrm{eV}$. However, one encounters an immediate obstacle: the Standard Model neutrino cross section is down from that of an electromagnetic or hadronic interaction by six orders of magnitude. This implies a low air shower rate and an accumulation of events at a low altitude where the target density is highest. On the other hand, the neutrino primary hypothesis is supported by the observed clustering. A couple of solutions to the small cross section problem have been proposed.

- Neutrino annihilation to Z-burst Our Universe if firmly believed to be filled with $1.95 \mathrm{~K}$ relic neutrinos [46]. Observed Universe has a large scale structure of superclusters and clusters. Sources for extreme energy neutrinos have been proposed in several models [47]. If the neutrino is massive, the CNB may be clustered in neutrinos clusters in our neighbourhood. If the primary particles which propagate across cosmic distances are neutrinos which then annihilate with the CNB within the GZK zone they will create a local flux of nucleons and photons above $E_{\mathrm{GZK}}$.

Since the $\mathrm{Z}$ burst annihilation process is resonant (see fig. 10), the event energy is unique and it is $E=4 \cdot 10^{21}\left(m_{\nu} c^{2} / \mathrm{eV}\right)^{-1} \mathrm{eV}$. The nucleons and photons are lower in energy by the multiplicity factor $(\sim 30)$. The strength of the Z-burst hypothesis is that the predicted high-energy for air-showers is in the range $10^{20}$ to $10^{22} \mathrm{eV}$ if we use the neutrino mass inferred from oscillations experiments as input parameter.

The Z-burst makes several predictions. The most relevant being:

(a) a significant flux of neutrinos;

(b) a $\gamma / p$ primary ratio of order 10 ;

(c) events pointing to sources; (d) pairing of events;

(e) pileup of cosmic rays from the typical Z-burst at $10^{19-20} \mathrm{eV}$;

(f) relationship maximum shower energy - neutrino mass.

- Strong $\nu$ cross section at $E \geq E_{\mathrm{GZK}}$ Limits on the strength of neutrino cross section at $10^{20} \mathrm{eV}$ can be inferred from existing data. The idea that neutrinos may have a strong interaction at high energy scale is not new. [48] However, three recent ideas concerning new interactions relate well to the $10^{20} \mathrm{eV}$ scale. The first idea is that leptons are bound state of dual QCD gluons [49] that reveal themselves above the electroweak scale $\sim \mathrm{TeV}$. The second idea is that Grand Unification occurs precouciously at $\sim \mathrm{TeV}$ because of extra dimensions and a neutrino above the threshold becomes strongly interacting [50]. The third idea is that the exchange of a towers of Kaluza-Klein modes from extra compactified dimensions lead to a strong neutrino cross section above $\sim \mathrm{TeV}$. The new threshold would have consequences for cross sections at lower energies, though not dramatic. Signatures for the three models include directional pointing to sources, shower profiles different from those of a proton or a gamma, and strong correlation observed energy - zenith angle.

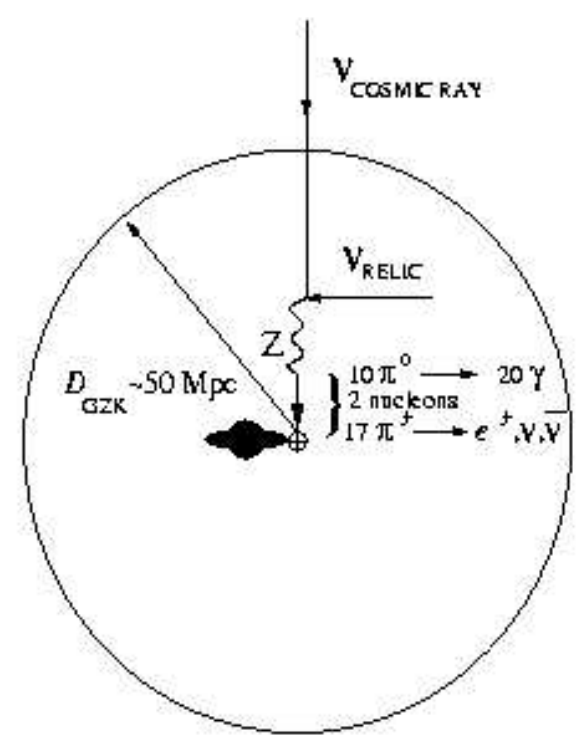

Fig. 10. Production of a Z-burst resulting from the resonant annihilation of a cosmic ray neutrino on a relic antineutrino (from Weiler [64]). 


\section{Lorentz Invariance (LI)}

Numerous solutions have been proposed for the EECRs paradox. As we have seen, low energy photons should interact with high energy particles. The reactions should take place because when Lorentz transformed to the center of mass frame the low energy photon has sufficient energy to overcome an intrinsic threshold. There is one mechanism that could resolve the paradox: a mechanism based on a simple, however drastic, assumption of a violation of ordinary Lorentz invariance.

In this case, for protons with energies $10^{20}$ and higher the center of mass energy is not enough for pion production in scattering with CMB photons. The GZK cut-off is deleted (or delayed).

With the idea of spontaneus symmetry breaking in particle physics came the suggestion that Lorentz invariance might be weakly broken at high energies [51]. It was suggested that LI may be broken as a consequence of a (new) theory of quantum gravity. A very tiny amount of LI symmetry breaking is required to avoid GZK by supressing the photomeson interactions between extreme energy protons and the CBR. Of course this would also eliminate any pile-up structure.

We will start by considering a class of dispersion relations which takes the form:

$$
E^{2}-p^{-2}-m^{2} \simeq \eta E^{2}\left(\frac{E}{E_{p}}\right)^{\alpha} \simeq \eta p^{-2}\left(\frac{E}{E_{p}}\right)^{\alpha}
$$

where $c=1$ and $m$ is the mass, $E$ is the energy and $p$ is the 3 -component momentum of the particle, $E_{p}\left(\sim 10^{22}\right) \mathrm{MeV}$ is the Planck energy scale and $\alpha$ and $\eta$ are free parameters that characterize the deviation from ordinary Lorentz invariance.
Relevant to our phenomenological considerations is the process in which the head-on collision between a soft photon $(\epsilon, q)$ and a high energy particle $\left(E_{1}, p_{1}\right)$ leads to the production of two particles $\left(E_{2}, E_{3}, p_{2}, p_{3}\right)$. The threshold condition for the Lorentz invariant case is:

$$
p_{1 . t h} \simeq \frac{\left(m_{2}+m_{3}\right)^{2}-m_{1}^{2}}{4 \epsilon}
$$

while considering the deformation introduced in 6.2 we get:

$$
\begin{aligned}
p_{1 . t h} & \simeq \frac{\left(m_{2}+m_{3}\right)^{2}-m_{1}^{2}}{4 \epsilon} \\
& +\eta \frac{p_{1, t h}^{2+\alpha}}{4 \epsilon E_{p}^{\alpha}}\left(\frac{m_{2}^{1+\alpha}+m_{3}^{1+\alpha}}{\left(m_{2}+m_{3}\right)^{1+\alpha}}-1\right) .
\end{aligned}
$$

As we can see, the threshold is delayed (or canceled if the process comes out to be forbidden). This could resolve the GZK problem. [52]

\section{IF THE GZK EFFECT IS CONSISTENT WITH DATA?}

The dullest possibility is that GZK is consistent with data. The strongest evidence for post-GZK comes from AGASA observations, while HiRes claimed to have found GZK cut-off. As said before, a recent simulations of De Marco, Blasi and Olinto [61] shows that a systematic error of $30 \%$ would make the two experiments to reasonably agree with each other, GZK feature presence still being uncertain. Also from Auger [62] no conclusive evidence for the absence of the GZK feature can be claimed so far. Better data are awaited.

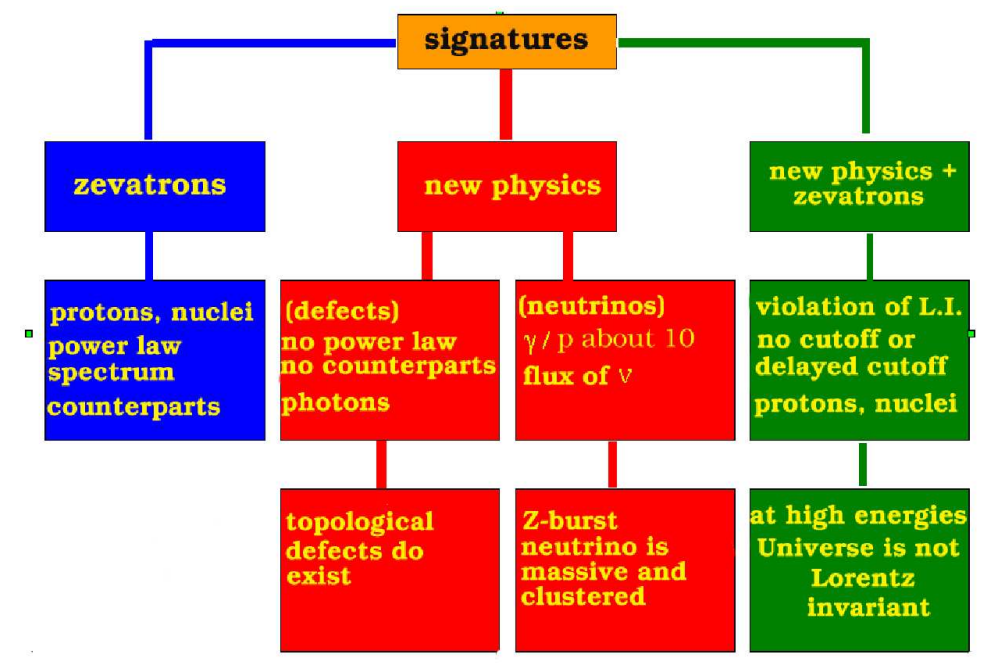

Fig. 11. A schematic diagram showing the most important signatures that characterize the most plausible models for EECRs origin 


\section{A. PAGLIARO}

\section{CONCLUSIONS AND DISCUSSION}

It is not too much to say that the current flood of papers and theories which try to explain and solve the mystery of Extreme Energy Cosmic Rays has produced a weird result: probably, the number of available theories are presently more than the number of events observed. This is a clear hint at a serious impasse in the scientific approach to the problem. Clearly and more wisely, the challenge should go back to the experimental arena. Future data will give us more clues relating to the origin of the post-GZK events.

Presently, we can only briefly summarize the clearest signature of the two different kind of theories (see fig.11). Main signatures for a bottom-up theory are:

- Protons/nuclei as primaries, with a much smaller number of neutrino-induced showers. The neutrinos being secondaries from photopion productions;

- A natural power law spectrum;

- Observation of counterparts;

- Events may cluster near the direction of sources;
Signatures for a top-down solutions are:

- Photons primaries. No heavy nuclei and more neutrinos than protons. [28] As a consequence, it would be more important to look deeper in the atmosphere for neutrino-induced showers (expected to be mostly horizontal);

- Non-power law spectrum. The spectra produced are much harder;

- No counterparts or repeats;

- A halo distribution of the events;

A complete absence of the GZK pile-up would be evidence for Lorentz invariance breaking. Saying more than this would be based on too weak evidence.

\section{ACKNOWLEDGMENTS}

Livio Scarsi introduced me to the fascinating mystery of cosmic rays. This paper is dedicated to his memory.
[1] K. Greisen, Phys. Rev. Lett. 16, 748 (1966).

[2] Z. T. Zatsepin, V. A. Kuzmin, J. Exp. Theor. Phys. Lett. 4, 78 (1966).

[3] A. W. Wolfendale, Adv. Space Res. 13, (12)687 (1993).

[4] D. J. Bird et al., Astrophys. J., Part 1 424, 491 (1994).

[5] M. Nagano, A. A. Watson, Rev. Mod. Phys. 72, 689 (2000).

[6] M. Takeda et al., Astrophys. J. 522, 225 (1999).

[7] N. Hayashida et al., Phys. Rev. Lett. 77, 1000 (1996).

[8] D. J. Bird et al., Phys. Rev. Lett. 71, 3401 (1993).

[9] T. Abu-Zayyad, et al., in Am. Phys. Soc. April Meeting, April 29-May 2, 2000 (Long Beach, CA), abstract C16.002 (2000).

[10] B. R. Dawson, R. Meyhandan, K. M. Simpson, Astropart. Phys. 9, 331 (1998).

[11] The Pierre Auger Collaboration, Science 318, 938 (2007).

[12] F. Halzen, R. A. Vazquez, T. Stanev, H. P. Vankov, Astropart. Phys. 3, 151 (1995).

[13] P. L. Biermann, Astron. Astrophys. 271, 649 (1993).

[14] R. Plaga, e-print arXiv:astro-ph/0111555 (2001).

[15] A. A. Penzias, R. W. Wilson, Astrophys. J. 142, 419 (1965).

[16] J. C. Mather et al., Astrophys. J., Part 1 420, 439 (1994).

[17] D. N. Spergel et al., Astrophys. J. 148, 175 (2003).

[18] J. L. Puget, F. W. Stecker, J. H. Bredekamp, Astrophys. J. 205, 638 (1976).

[19] M. Blanton, P. Blasi, A. V. Olinto, Astropart. Phys. 15, 275 (2001).

[20] R. J. Protheroe, e-print arXiv:astro-ph/9612212 (1996).

[21] M. G. Baring, in Very High Energy Phenomena in the Universe; Morion Workshop, edited by Y. GiraudHeraud, J. Tran Thanh Van, 97 (1997).

[22] A. M. Hillas, Ann Rev. Astron. Astrophys. 22, 425
(1984).

[23] A. Venkatesan, M. C. Miller, A. Olinto, Astrophys. J. 484, 323 (1997).

[24] J. P. Rachen, P. L. Biermann, Astron. Astrophys. 272, 161 (1993).

[25] P. L. Biermann, Space Sci. Rev. 74, 385 (1995).

[26] T. W. Jones, e-print arXiv:astro-ph/0012483 (2000).

[27] L. A. Anchordoqui, J. F. Beacom, H. Goldberg, S. Palomares-Ruiz, T. J. Weiler, Phys. Rev. D 75, 063001 (2007); e-print arXiv:astro-ph/0611581 (2006).

[28] F. A. Aharonian, the HEGRA Collaboration, in $A b$ stracts of the 19th Texas Symposium on Relativistic Astrophysics and Cosmology, held in Paris, France, Dec. 14-18, 1998, edited by J. Paul, T. Montmerle, E. Aubourg (CEA Saclay, 1998).

[29] F. A. Aharonian, the HEGRA Collaboration, Astron. Astrophys. 393, L37 (2002); e-print arXiv:astro$\mathrm{ph} / 0207528$ (2002).

[30] F. A. Aharonian et al., e-print arXiv:astro-ph/0703427 (2007).

[31] A. Bykov, Space Sci. Rev. 99, 317 (2001).

[32] V. Antonuccio, A. Pagliaro, C. Ferrigno, Frontier Objects in astrophysics and particle physics $\mathbf{8 5}$, (2002).

[33] R. P. Kirshner, A. Oemler, Jr., P L. Schechter, S. A. Shectman, Astrophys. J., Part 2 248, L57 (1981).

[34] F. W. Stecker, M. H. Salamon, Astrophys. J. 512, 521 (1999).

[35] M. Ave, R. A. Vazquez, E. Zas, J. A. Hinton, A. A. Watson, Astropart. Phys. 14, 109 (2000).

[36] F. Halzen, R. A. Vazquez, T. Stanev, H. P. Vankov, Astroparticles Phys. 3, 151 (1995).

[37] G. Sigl, D. N. Schramm, P. Bhattacharjee, Astropart. Phys. 2, 401 (1994).

[38] C. T. Hill, D. N. Schramm, Phys. Rev. D 31, 564 (1985). 
[39] P. Bhattacharjee, N. C. Rana, "Ultrahigh-energy particle flux from cosmic strings," ADS 1990STIN...9022525B.

[40] P. Bhattacharjee, G. Sigl, Phys. Rev. D 51, 4079 (1995).

[41] R. H. Brandenberger, Int. J. Mod. Phys. A 2, 77 (1987).

[42] V. Berezinsky, X. Martin, A. Vilenkin, Phys. Rev. D 56, 2024 (1997).

[43] V. Berezinsky, A. Vilenkin, Phys. Rev. Lett. 79, 5202 (1997).

[44] L. Masperi, G. Silva, Astropart. Phys. 8, 173 (1998).

[45] V. A. Kuzmin, V. A. Rubakov, Phys. Atomic Nucl. 61, 1028 (1998).

[46] B. W. Lee, S. Weinberg, Phys. Rev. Lett. 39, 165 (1977).

[47] V. Berezinsky, M. Kachelriess, A. Vilenkin, in Abstracts of the 19th Texas Symposium on Relativistic Astrophysics and Cosmology, held in Paris, France, Dec. 1418, 1998, edited by J. Paul, T. Montmerle, E. Aubourg (CEA Saclay, 1998).

[48] V. S. Berezinsky, G. T. Zatsepin, Phys. Lett. B 28, 423 (1969).

[49] J. Bordes, Hong-Mo Chan, J. Faridani, J. Pfaudler, Sheung Tsun Tsou, Astropart. Phys. 8, 135 (1998).

[50] G. Domokos, S. Kovesi-Domokos, Paul T. Mikulski, eprint arXiv:hep-ph/0006328 (2000).

[51] H. Sato, T. Tati, Prog. Theor. Phys. 47, 1788 (1972).

[52] L. Gonzalez-Mestres, in Workshop on Observing Giant Cosmic Ray Air Showers From >10(20) eV Particles From Space: College Park, Maryland, November, 1997, edited by J. F. Krizmanic, J. F. Ormes, R. E. Streitmat- ter (Woodbury, New York, AIP Conference Proceedings 433, 1998), p. 148.

[53] G. B. Thomson, e-print arXiv:astro-ph/0609403 (2006).

[54] K. Kobayakawa et al. Proc. 26th Int. Cosmic Rays Conf. 4, 467 (1999).

[55] M. Catanese et al., Astrophys. J. 469, 572 (1996).

[56] O. V. Zurhenkov, A. V. Plyasheshnikov, Nucl. Phys. B 75, 296 (1999).

[57] K. Bernlohr et al., Astropart. Phys. 8, 253(1998).

[58] S. P. Swordy, D. B. Kieda, Astropart. Phys. 13, 137 (2000).

[59] T. K. Gaisser, J. Phys.: Conf. Ser. 47, 15 (2006).

[60] J. H. MacGibbon, R. H. Brandenberger, Nucl. Phys. B 331, 153 (1990).

[61] D. Di Marco, P. Blasi, A. V. Olinto, Astropart. Phys. 20, 53 (2003).

[62] P. Sommers et al., the Pierre Auger Collaboration, in Proceedings of the 29th International Cosmic Rays Conference (Pune, India, 2005).

[63] J. M. Knerr, J. R. Jokipii, D. C. Ellison, Astrophys. J. 458, 641 (1996).

[64] T. J. Weiler, in Radio Detection of High Energy Particles, First International Workshop RADHEP 2000, held in Los Angeles, California, 16-18 November, 2000, edited by D. Saltzberg, P. Gorham, AIP Conference Proceedings 579, (Melville, NY, American Institute of Physics, 2001), p. 58.

\title{
ЗАГАДКА ВИСОКОЕНЕРГЕТИЧНИХ КОСМІЧНИХ ПРОМЕНІВ
}

\author{
А. Пальяро \\ Інститут космічної фізики й астрофізики, Палермо, Італія
}

\begin{abstract}
Після відкриття космічного мікрохвильового фону було показано, що космічні промені високих енергій повинні бути суттєво ослабленими. Незважаючи на це, зареєстровано кілька подій. Джерела цих космічних променів і механізми їх утворення дотепер залишаються таємницею, і непевні свідчення про обрізання, пов'язане з космічним фоном, є на сьогодні головним викликом. Виглядає на те, що бракує якогось фундаментального факту в наших уявленнях про природу та поширення космічних променів і про їхні джерела. Як можуть бути ці частинки настільки прискореними чи утвореними на таких енергіях? Які джерела відповідальні за це? Оскільки походження променів, імовірно, позагалактичне, то як вони можуть долати космологічні віддалі? У цій статті показано, чому ці питання дотепер без відповіді і які пояснення поки що є найправдоподібнішими.
\end{abstract}

